\title{
Exercise during pregnancy activates cardio-protective genes without a further increase in pregnancy-induced cardiac hypertrophy.
}

\author{
Eunhee Chung ${ }^{1 *}$, Kalli D Looten², Taylor Lunsford ${ }^{2}$, Tracer Skelton², Katherine A Grue ${ }^{2}$ \\ ${ }^{1}$ Department of Kinesiology, Health and Nutrition, University of Texas at San Antonio, San Antonio, TX, USA \\ ${ }^{2}$ Department of Kinesiology and Sport Management, Mail Box 43011, Texas Tech University, Lubbock, USA
}

\begin{abstract}
Exercise and pregnancy are known to induce physiological cardiac hypertrophy, and the molecular mechanisms have recently been revealed exercise-induced and pregnancy-induced cardiac hypertrophy. However, current understanding of the interaction of exercise and pregnancy on heart health is poorly understood. Our objectives were to study how exercise during pregnancy modifies pregnancy-induced cardiac hypertrophy and to determine gene expression patterns when two different physiological hypertrophic stimuli are concurrent. Female C57BL/6 mice were divided into three groups: non-pregnant sedentary control (C), sedentary pregnant (LP), and exercise during pregnant (ExP) groups. The mice in the ExP group were exercised voluntarily from gestational day 1 through gestational day 17, at which time they were sacrificed. Both the ExP and LP groups showed a significant increase in heart mass compared to the $C$ group. Phosphorylation of Akt, mTOR, and p70S6K were all significantly increased in the hearts of the ExP group when compared to the $C$ group. Phosphorylation of mTOR was different between the ExP and the LP groups. The ExP group displayed significant upregulation of $\alpha$-myosin heavy chain compared to the LP group. In addition, the ExP group significantly downregulated $\mathrm{Ccl} 2$ (monocyte chemotactic protein) compared to the $\mathrm{C}$ group. Taken together, our results demonstrate that exercise during pregnancy initiated at day one of gestation may be beneficial by activating several cardiac protective genes.
\end{abstract}

Keywords: Exercise during pregnancy, Cardiac hypertrophy, Gene expression.

Accepted on April 10, 2017

\section{Introduction}

Pregnancy and exercise promote cardiovascular remodeling [1]: pregnancy is associated with prolonged, but reversible, cardiac volume overload secondary to increased blood volume [2] and significant hormonal changes that together result in cardiac hypertrophy $[3,4]$. During pregnancy, cardiac output increases gradually and reaches a peak from the secondtrimester through term [5]. This increase in cardiac output is achieved by either gradual and substantial increases in heart rate and stroke volume or a fall in vascular resistance [5]. In addition to hemodynamic alterations during pregnancy, the levels of circulating progesterone gradually increase with advancing gestational age, which contributes to pregnancyinduced cardiac hypertrophy $[4,6]$. Similar to pregnancy, exercise training increases cardiac output. However, increased cardiac output in response to exercise training occurs mainly through increased stroke volume. In addition, both the volume overload and increased heart rate are intermittent in exercise rather than continuous as in pregnancy.

Cardiac growth in response to pregnancy and exercise is beneficial to the heart and called physiological cardiac hypertrophy in contrast to pathological cardiac hypertrophy, which is typically induced by pressure or volume overload $[1,7]$. Several criteria have been used to distinguish these two different types of cardiac hypertrophy. Pathological cardiac hypertrophy is often accompanied by induction of "fetal gene program", increased fibrosis, imbalance between angiogenesis and cardiac muscle mass and cardiac dysfunction, while physiological cardiac hypertrophy does not follow these patterns [1]. In addition, accumulated evidence from transgenic and knockout animal models suggest that signaling pathways underlying physiological cardiac hypertrophy are distinct from those induced by pathological stimuli $[8,9]$.

Akt and its downstream targets (i.e., GSK3, mTOR, and p70S6K) play an important role in physiological cardiac hypertrophy $[4,8,10]$. The active phosphorylated form of Akt has been shown to increase after swim training [9], voluntary wheel running [11], and during pregnancy $[4,10]$ in mice. However, the time course of Akt activation differs between exercise and pregnancy stimulation. The level of phosphorylation of Akt is elevated in early exposure to exercise training and is attenuated gradually to the level of sedentary control $[9,11]$. In contrast, the phosphorylation of Akt is increased in midpregnancy and sustained until the late pregnancy stage [4]. The signaling pathways underlying cardiac hypertrophy when both physiological stimuli (i.e., pregnancy and exercise) are present are currently poorly understood.

Although pregnancy-induced cardiac hypertrophy is recognized as physiological, the number of women who develop heart problems during pregnancy is increasing [12,13]. Previous studies have shown that late pregnancy is associated with mild impairment of left ventricular diastolic [14] and systolic function [3,4]. In addition, the heart during late pregnancy is more vulnerable to ischemia/reperfusion injury [15] and could result in an adverse event such as peripartum cardiomyopathy 
Citation: Chung E, Looten KD, Lunsford T, et al. Exercise during pregnancy activates cardio-protective genes without a further increase in pregnancy-induced cardiac hypertrophy. Gynecol Reproduct Endocrinol -UK. 2017;1(1):7-15

$[10,16]$. Oxidative stress and the anti-angiogenic environment of late-pregnancy have, thus far, been suggested as mechanisms responsible for cardiac dysfunction and disease associated with pregnancy $[10,16]$. Exercise training, on the other hand, has been shown to increase cardiac angiogenesis [17], decrease inflammation [18], and increase antioxidant capacity [19]. These exercise adaptations may be critical for the hearts of late-pregnancy that is vulnerable to ischemia/reperfusion injury [15] and prone to oxidative stress, angiogenic imbalance, and inflammation $[10,16,20]$. The objectives of this study were to investigate how exercise during pregnancy modifies pregnancyinduced cardiac hypertrophy. In addition, gene expression that has been implicated in physiological and pathological cardiac hypertrophy was measured to characterize cardiac hypertrophy during pregnancy with exercise (i.e., angiogenesis, antioxidant, inflammation, and extracellular matrix).

\section{Materials and Methods}

\section{Animals}

Virgin female C57BL/6 mice were obtained from Charles River Laboratories at 10 weeks of age. Animals were housed in a temperature- and a light- controlled room with food and water available ad libitum throughout the study. All of the animals were handled and euthanatized under the approved protocol by the Texas Tech University Animal Care and Use Committee. Detail information for pregnancy, exercise protocol, and beneficial effects of maternal exercise on fetal heart adaptation was described in recent our publication [21]. Briefly, mice were divided into three groups ensuring no initial body weight differences among groups: non-pregnant sedentary control (C), sedentary pregnant (LP), and exercise during pregnant (ExP) groups. Mice were fasted for 4 hours in a sedentary cage before being euthanized in order to avoid the effects of acute exercise and feeding. Mice were euthanized by placing the mouse in an isoflurane-induction machine followed by cervical dislocation.

\section{Protein analyses}

Protein expression levels in the left ventricular tissues were evaluated using the standard Western blot method. Left ventricular tissues were homogenized in RIPA lysis buffer (Sigma) containing protease and phosphatase inhibitor singleuse cocktail (Thermo Scientific). Total protein concentration was determined using Pierce $660 \mathrm{~nm}$ according to the manufacturer's instructions (Thermo Scientific). Primary antibodies for Akt (Cell signaling, \#9272), phospho-Akt (Ser 473; Cell signaling, \#4060), Gsk3 $\beta$ (Santa Cruz, sc-7291), phospho-GSK3 $\beta$ (Ser 9; Santa Cruz, sc-11757), mTOR (cell signaling, \#2983), phospho-mTOR (Ser2448; cell signaling, \#2971), p70S6K (cell signaling, \#9272), and phospho-p70S6K (Thr421/Ser424; cell signaling, \#9204) were used. Detailed information about the Western blot method was described previously [4,21]. The degree of activity of corresponding proteins was determined by the ratio of phospho to total protein. Four to five animals per group were used with four to five technical replicates per animal, and a representative blot was shown.

\section{$m R N A$ analyses}

Total RNA from left ventricular tissues was isolated using a Tri Reagent kit according to the manufacturer's instructions
(Molecular Research Center, Inc., Cincinnati, OH). Detailed information for total RNA isolation and cDNA synthesis was described previously [21]. The levels of all candidate genes were normalized to Hprtl and results were plotted as fold changes relative to the $\mathrm{C}$ group. Primers for Myh6

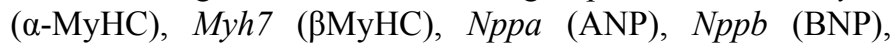
Acta1 ( $\alpha$-skeletal actin), Atp2b (SERCA2), collagen type 1 alpha 1 (Colla1), Col3a1, Col8a1, connective tissue growth factor (Ctgf), angiopietin-1 (Angpt1), Angpt2, fibroblast growth factor $2(F g f 2)$, platelet derived growth factor alpha $(P d g f a)$, peroxisome proliferator-activated receptor gamma coactivator-1 alpha (Ppargcla), vascular endothelial growth factor a $(V e g f a)$, metallothionein $2(M t 2)$, signal transducer and activator of transcription 3 (Stat3), mitochondrial superoxide dismutase $(\operatorname{Sod} 2)$, and monocyte chemo attractant protein-1 $\left(\mathrm{Ccl}_{2}\right)$ listed previously [4,20,22,23]. Additional primers were, carnitine palmitoyl transferase 1B, muscle, (Cpt $1 b)$, forward 5'-TTCACTGTGACCCCAGACGGG-3', reverse 5'-AATGGACCAGCCCCATGGAGA-3'; mediumchain acyl-coenzyme A dehydrogenase (Acadm), forward 5'-TCGAAAGCGGCTCACAAGCAG-3', reverse 5'CACCGCAGCTTTCCGGAATGT-3'; Tumor necrosis factor alpha(Tnfa), forward 5'-GTCTCAGCCTCTTCTCATTCCT-3', reverse 5'-GTCTTTGAGATCCATGCCGT-3'; interleukin-6 (ILO), forward 5'-ACCAGAGGAAATTTTCAATAGGC-3', reverse 5'-TGATGCACTTGCAGAAAACA-3'. As many studies report fetal gene programs with their known name, we did not use the official gene name for the following mRNAs: $\alpha$ MyHC, $\beta$-MyHC, ANP, BNP, $\alpha$-skeletal actin, and SERCA2A. Other mRNAs were written according to the guidelines for formatting mouse gene name (http://www.biosciencewriters. com/Guidelines-for-Formatting-Gene-and-Protein-Names. aspx): italicized with only the first letter in upper-case (i.e., $\mathrm{Ccl}_{2}$ ). Protein symbols of mice are not italicized and all letters are in upper case (i.e., $\mathrm{CCL}_{2}$ )

\section{Statistical analysis}

All results are expressed as mean \pm SEM. Statistical significance was tested by one-way analysis of variance (ANOVA) with post hoc Tukey's test. $\mathrm{p}<0.05$ was regarded as significant between groups. Statistical analyses were performed using GraphPad Prism version 6 software.

\section{Results}

\section{Effects of exercise during pregnancy}

The phenotypes of pregnant dams, including the pattern of running activity, gestational weight gain, average fetal body weight, and the number of pubs were reported previous publication [21]. Briefly, the pattern of running activity was high in early pregnancy and gradually decreased to low levels after mid-pregnancy [21]. In agreement with previous studies $[3,4]$, pregnancy did induce cardiac hypertrophy. Both absolute heart weight and heart weight (HW) normalized to tibia length (TL) (Figure 1), were significantly higher in the ExP and the LP groups when compared with the $\mathrm{C}$ group $(\mathrm{p}<0.05)$. The percent increase in HW/TL was $21.1 \pm 1.9 \%$ in the ExP and $17.6 \pm 4.5 \%$ in LP compared with C. However, the ExP group did not have significantly greater $\mathrm{HW}$ or HW/TL when compared with the LP group ( $\mathrm{p}>0.05)$. 


\section{Akt signaling pathways in the heart from exercise during pregnancy}

The activity of Akt showed a significant increase in phosphorylation in the ExP and LP groups compared to the $C$ group $(p<0.05)$. However, there were no differences in the activity of Akt between the ExP and LP groups ( $p>0.05$; Figure $2 A)$. Phosphorylation of GSK3 $\beta$ was not altered in the ExP and LP groups compared to the $\mathrm{C}$ group ( $\mathrm{p}>0.05$; Figure $2 \mathrm{~B}$ ).
Phosphorylation of mTOR was significantly increased in the ExP group as compared to the $\mathrm{C}$ group $(\mathrm{p}<0.05)$, and significantly different between the ExP and the LP groups $(p<0.05$; Figure $2 \mathrm{C})$. Phosphorylation of p70S6K was significantly increased in both ExP and LP compared to $\mathrm{C}(\mathrm{p} \leq 0.01$; Figure 2D).

\section{$m R N A$ profiles of the hearts from exercise during pregnancy}

We characterized cardiac hypertrophy in response to exercise

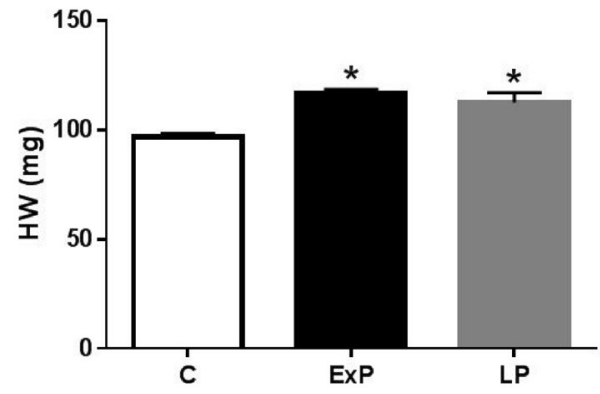

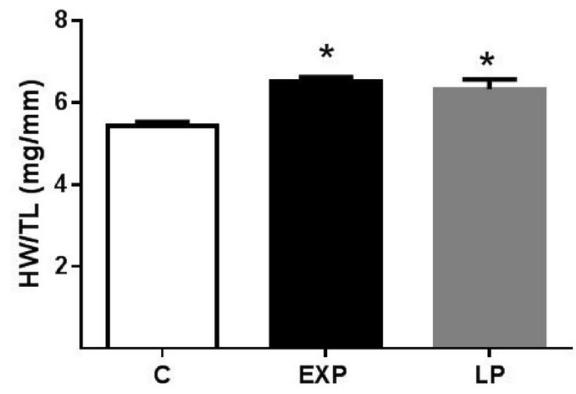

Figure 1: Cardiac hypertrophy in response to exercise during pregnancy and pregnancy alone. A) Absolute heart weight and B) Heart weight (HW) normalized to tibia length (TL), was significantly higher in the ExP and LP when compared to C. C: non-pregnant sedentary control; LP: sedentary pregnant group; ExP: exercise during pregnancy. Values are expressed by mean $\pm S E M$. ${ }^{*} p<0.05$, significantly different from $C$.
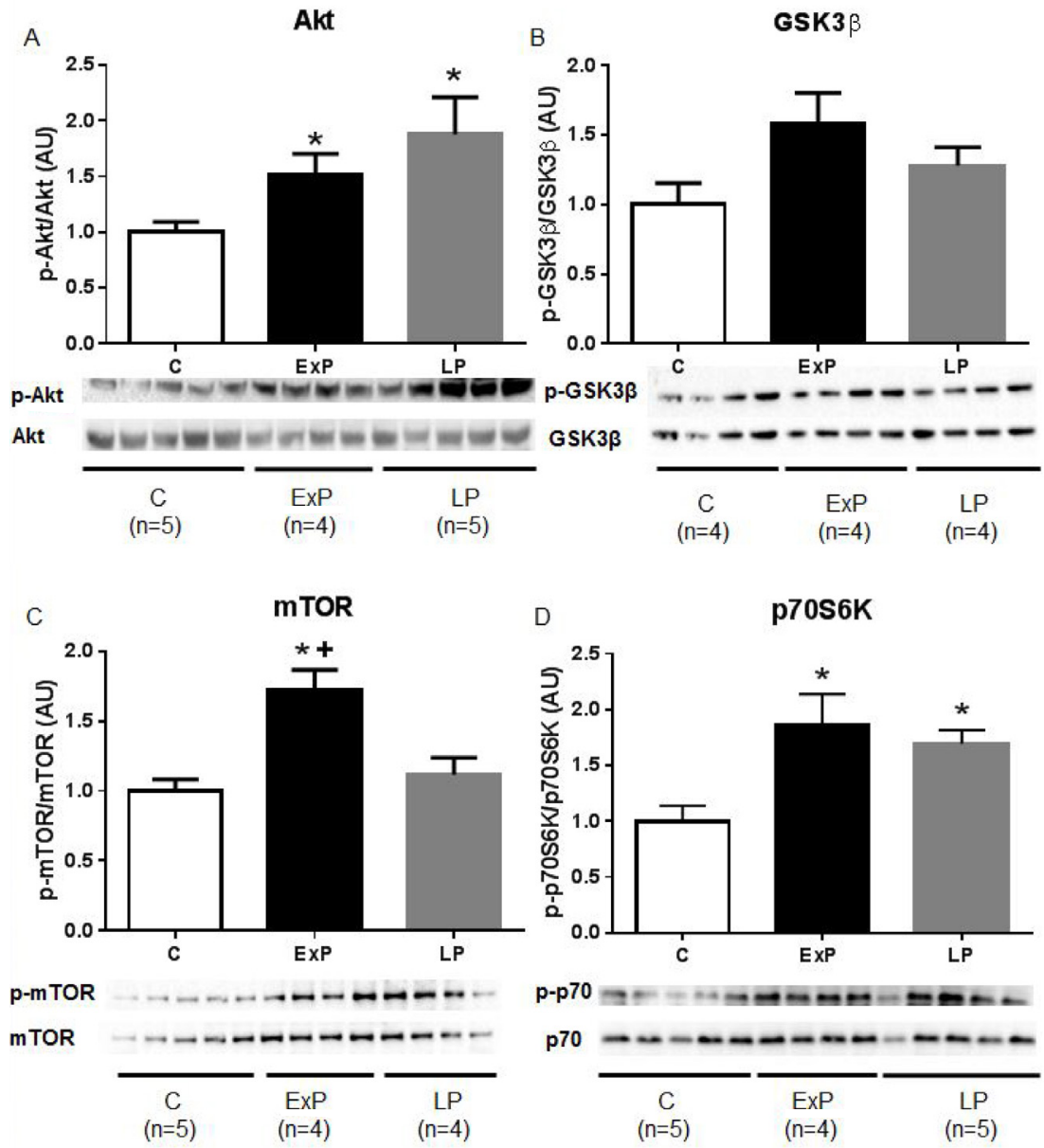

Figure 2. Akt and its downstream targets in response to exercise during pregnancy. A) Akt phosphorylation was significantly increased in the ExP and LP as compared to the C group. B) GSK3 $\beta$ phosphorylation was not altered in the ExP and LP as compared to the C group. C) $m$ TOR phosphorylation was significantly increased in ExP compared to C and LP. D) p70S6K phosphorylation was significantly increased in both ExP and LP. C: non-pregnant sedentary control; ExP: exercise during pregnancy; LP: sedentary pregnant group. Values are expressed by mean \pm $S E M .{ }^{*} p<0.05$, significantly different from $C ;+p<0.05$, significantly different from $L P$. 
Citation: Chung E, Looten KD, Lunsford T, et al. Exercise during pregnancy activates cardio-protective genes without a further increase in pregnancy-induced cardiac hypertrophy. Gynecol Reproduct Endocrinol -UK. 2017;1(1):7-15

during pregnancy by measuring gene expression patterns that are important for cardiac remodeling (Figures 3-6). In agreement with previous studies [4,24], we found that fetal genes that are typically associated with pathological hypertrophy were not altered in LP (Figure 3). $\alpha$-MyHC and ANP were significantly upregulated in the $\operatorname{ExP}(\mathrm{p}<0.05)$, while $\beta$-MyHC, BNP, $\alpha$-skeletal actin, and SERCA2A were not altered compared to the $\mathrm{C}$ group ( $p>0.05$; Figure 3 ). The expression of $\alpha-\mathrm{MyHC}$ was significantly upregulated in the ExP group when compared to the LP group $(p<0.05$; Figure 3$)$. Further, we measured genes involved in mitochondrial fatty acid oxidation due to pathological cardiac hypertrophy being characterized by a metabolic switch from fatty acid oxidation to glycolysis [25]. The expression of Cpt1b was significantly upregulated in the ExP group compared to the $C$ group $(\mathrm{p}<0.05$; Figure 3$)$, while Acadm was not altered among groups. We measured genes associated with antioxidant defense system and markers of inflammation (Figure 4). Mt 2 was significantly upregulated in the ExP and LP groups compared to $\mathrm{C}(\mathrm{p}<0.05)$. There were no differences seen in Stat 3 and Sod 2 in the ExP and LP groups compared to the $C$ group ( $p>0.05$; Figures $4 \mathrm{~A}-4 \mathrm{C}$ ). In addition, we found that $\mathrm{Ccl}_{2}$ was not altered in the LP group ( $p>0.05)$, but significantly downregulated in the ExP group compared to the $C$ group $(p<0.001$; Figure 4D). Tnfa and IL6 were not changed in the ExP and LP groups compared to the $C$ group ( $p>0.05$ : Figures $4 \mathrm{E}$ and $4 F$ ).

Next, we measured mRNA levels of collagen (Figure 5) and angiogenic factors (Figure 6). mRNA levels of collagens, including Colla1, Col3al, and Col8al were not altered in the ExP and LP groups compared to the C group ( $\mathrm{p}>0.05)$. Ctgf
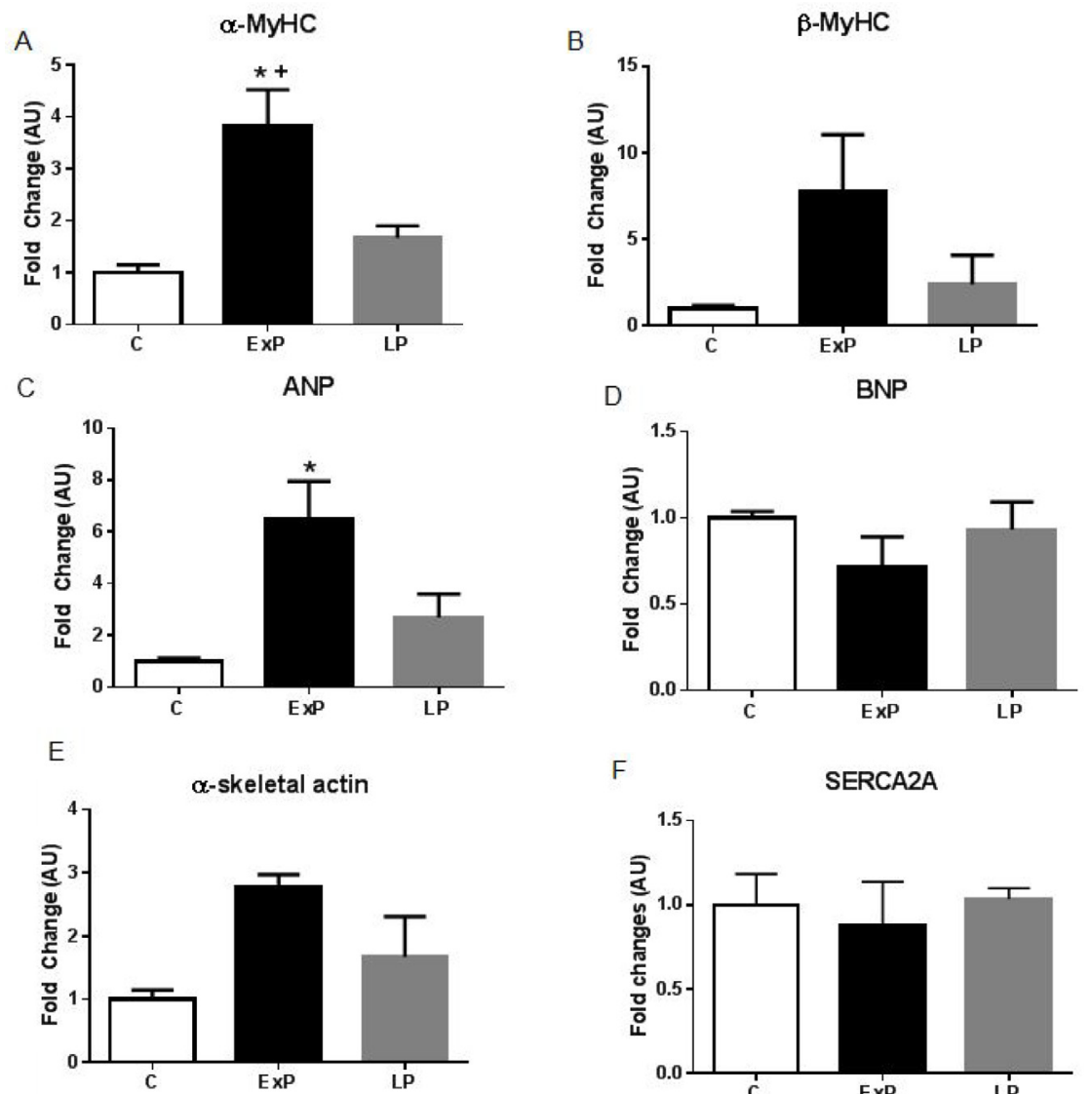

F
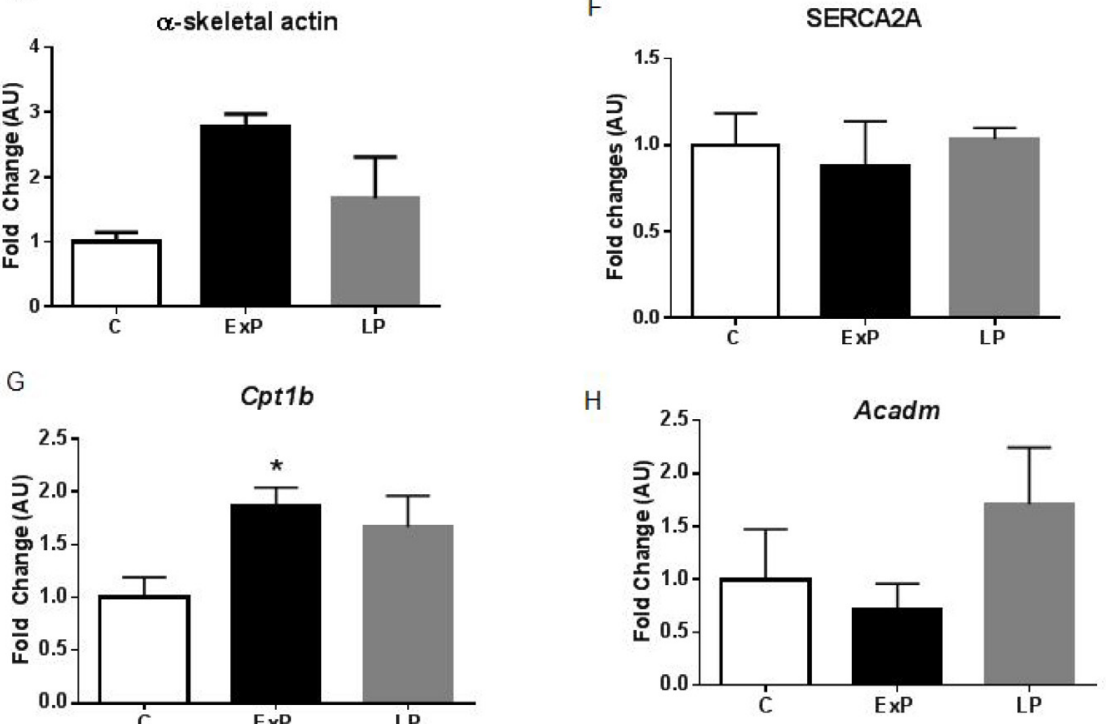

Figure 3. $m R N A$ levels of genes associated with pathological hypertrophy. MyHC: myosin heavy chain; ANP: atrial natriuretic peptide; BNP, brain natriuretic peptide; and SERCA2A, cardiac Ca2+-ATPase of the sarcoplasmic reticulum; Cpt1b: muscle carnitine palmitoyltransferase 1B; Acadm: medium-chain acyl-CoA dehydrogenase. C: non-pregnant sedentary control; ExP: exercise during pregnancy; LP: sedentary pregnant group. Values are expressed by mean \pm SEM expressed as fold change relative to $C .{ }^{*} p \leq 0.05$, significantly different from $C ;+p<0.05$, significantly different from $L P$. 
was significantly decreased in the ExP and in the LP groups compared to the $\mathrm{C}$ group ( $\mathrm{p}<0.05$; Figure 5$)$. Further, genes regulating angiogenic factors, including Ppargcla, Vegfa, Angt1, Angt2, and Fgf2 were not altered in the ExP and LP groups compared to the $\mathrm{C}$ group ( $\mathrm{p}>0.05$; Figure 6). Pdgfa was significantly upregulated in the ExP group compared to the $\mathrm{C}$ group ( $\mathrm{p}<0.05)$, but $P d g f a$ was not significantly changed in the LP group ( $\mathrm{p}>0.05$ ) compared to the $\mathrm{C}$ group.

\section{Discussion}

Exercise during pregnancy initiated at day one of gestation does not augment pregnancy-induced cardiac hypertrophy. Although it may not be sufficient enough to additionally activate signaling molecules and genes associated with pregnancy-induced cardiac hypertrophy, maternal exercise during pregnancy seems not be detrimental for mothers. The Akt signaling pathway was increased during pregnancy with or without the addition of gestational exercise. Interestingly, phosphorylation of mTOR is significantly different between the ExP and LP groups. Transcriptional profiles of the heart in response to exercise during pregnancy (Figures 3-6) displayed many of the features of physiological hypertrophy: 1) upregulation of $\alpha$-MyHC and ANP (Figures 3A and 3C) which is highly correlated to an increase in contractile properties 2) upregulation of Cpt1b (Figure 3G), a rate-limiting enzyme in the mitochondria responsible for the transport of long chain fatty acid; and, 3) downregulation of $\operatorname{Ctg} f$ (Figure 5D), a connective tissue growth factor which suggests no induction of fibrosis in the heart of the ExP animals. However, most of the findings were not significantly different between ExP and LP groups, this may
A

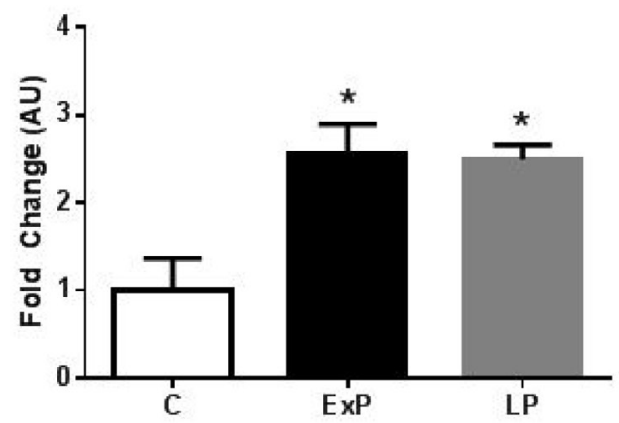

C

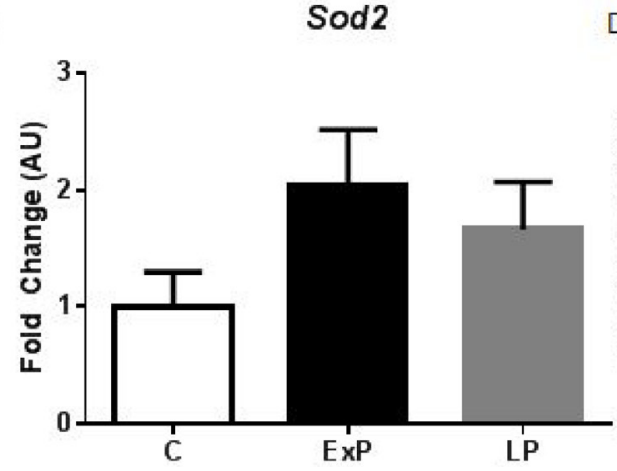

E

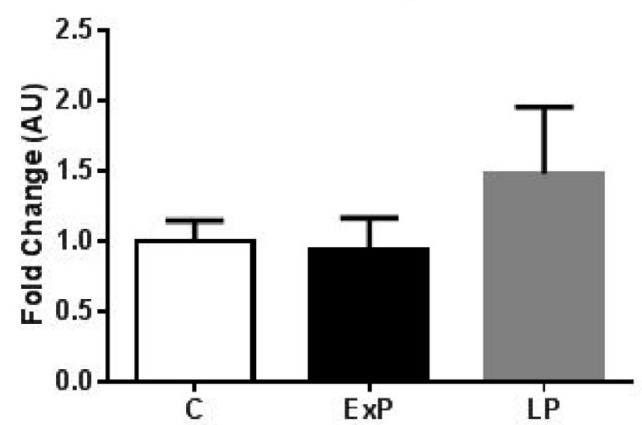

B

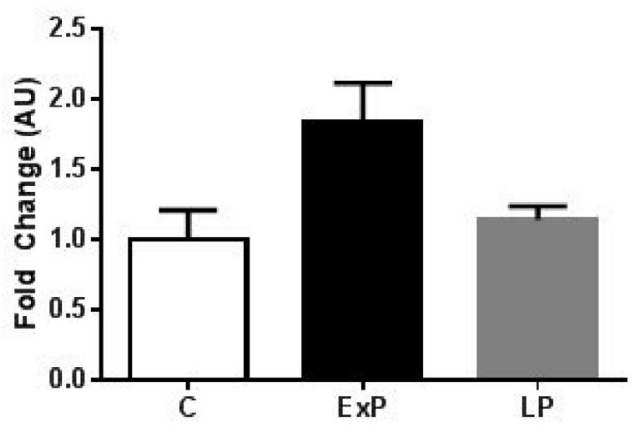

D

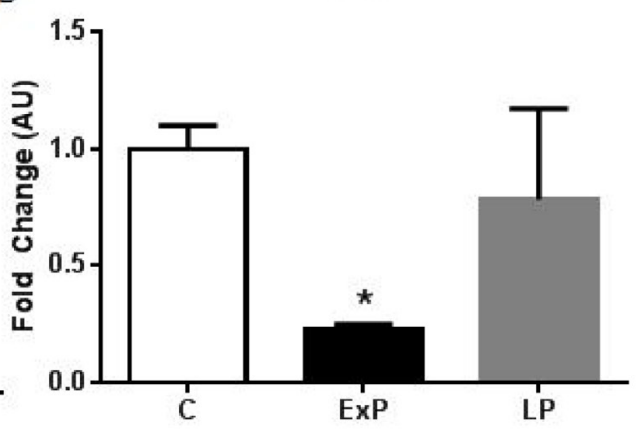

F

IL6

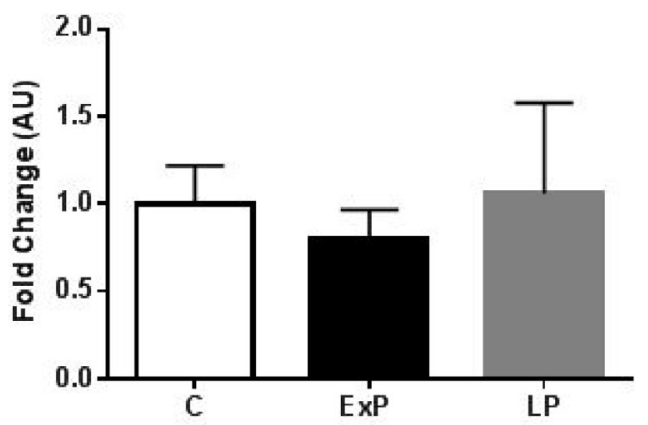

Figure 4. mRNA levels of genes associated with antioxidant properties and inflammation. Mt2: metallothionein 2; Stat3: signal transducer and activator of transcription 3; Sod2: mitochondrial superoxide dismutase; Ccl2: monocyte chemoattractant protein-1; Tnfa: Tumor necrosis factor alpha; IL6: interleukin-6. C: non- pregnant sedentary control; ExP: exercise during pregnancy; LP: sedentary pregnant group. Values are expressed by mean $\pm S E M$ expressed as fold change relative to $C$. ${ }^{*} p<0.05$, significantly different from $C$. 
Citation: Chung E, Looten KD, Lunsford T, et al. Exercise during pregnancy activates cardio-protective genes without a further increase in pregnancy-induced cardiac hypertrophy. Gynecol Reproduct Endocrinol -UK. 2017;1(1):7-15
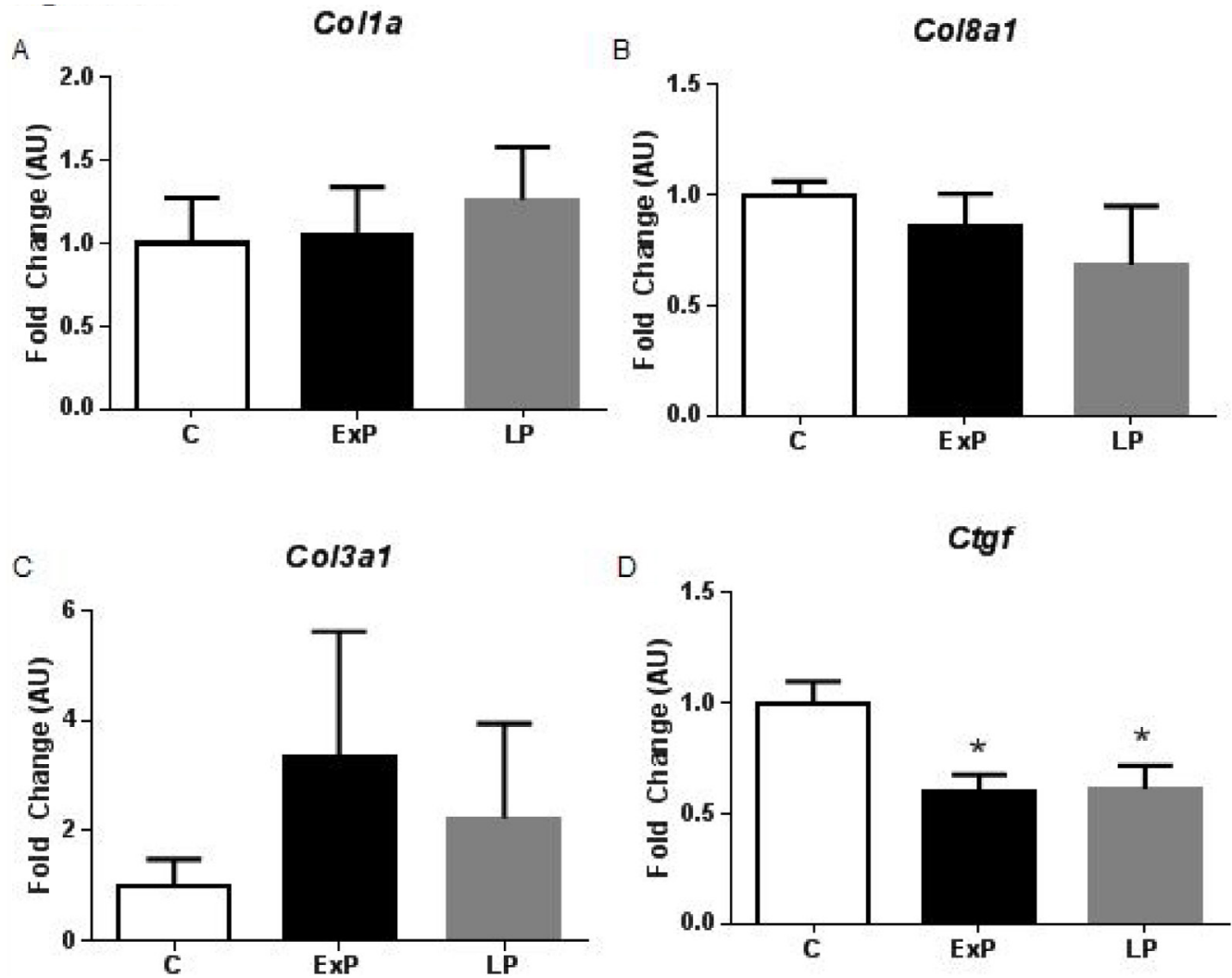

Figure 5. mRNA levels of genes associated with extracellular matrix (A-D). Colla1: collagen type 1 alpha 1; Col3a1: collagen type 3 alpha 1; Col8a1: collagen type 8 alpha 1; Ctgf: connective tissue growth factor. C: non-pregnant sedentary control; ExP: exercise during pregnancy; LP: sedentary pregnant group. Values are expressed by mean $\pm S E M$ expressed as fold change relative to $C .{ }^{*} p<0.05$, significantly different from $C$.

indicate that gestational exercise does not further stimulate the pregnancy-associated cardiac adaptation.

Our results (Figure 2) indicate that Akt and its downstream targets are important mediators of cardiac hypertrophy in response to exercise during pregnancy. The phosphorylation of Akt, mTOR, and p70S6K were all increased in the ExP group, which has been shown in non-pregnant animals after exercise training $[11,26,27]$. Our findings are consistent with previous pregnancy studies showing that the phosphorylation of Akt is upregulated in the LP group [4,10], and phosphorylation of GSK3 $\beta$ and mTOR were not altered in this group [4]. In contrast to a previous study [4], we found that the phosphorylation of p70S6K was significantly increased in the LP group. The discrepancy between the previous study [4] and our current study may be due to the difference in gestational age. The previous study sacrificed animals at 18-19 days of gestation when estrogen levels are high and progesterone levels begin to drop (1-2 days before parturition); the current study examined tissues at 17 days of gestation, when progesterone levels are high [1]. Although we showed the equivalent phosphorylation of p70S6K in the ExP and LP groups, phosphorylation of mTOR was significantly higher in the ExP group compared to the LP group. Thus, there must be another upstream molecule that can regulate $\mathrm{p} 70 \mathrm{~S} 6 \mathrm{~K}$ [28].

Induction of the fetal gene program is generally used to distinguish between physiological and pathological cardiac hypertrophy [1]. There are still confounding results regarding the fetal gene expression in response to exercise. Some studies report that exercise training increases $\alpha$-MyHC [26,29] and
ANP [30], which agreed with our results (Figure 3). However, others show no changes in these genes (see a comparison among studies in the review article published [24]). In addition, fibrosis is known to occur due to pathological stimuli and is used to differentiate between physiological and pathological hypertrophy [1]. Previous studies show that there is no induction of fibrosis in the heart in response to exercise training [9] and pregnancy $[3,4]$, while hearts from pathological stimuli display an increase in fibrosis [9]. Our results (Figure 5) suggest that exercise during pregnancy does not upregulate mRNA levels of collagen, and significantly downregulates Ctgf in the ExP and LP animals. Thus, we regard that cardiac hypertrophy in response to exercise during pregnancy is not of a pathological nature and it does not appear to activate the pro-fibrotic genes. It has been suggested that increased cardiac inflammation, oxidative stress, and angiogenic imbalance during late pregnancy contribute to the development of pericardium cardiomyopathy $[10,20]$. In the transgenic mouse model, sustained activation of Akt with low STAT3 level accelerates cardiac inflammation and leads to cardiac myopathy during pregnancy by upregulating $C C L_{2}$ mRNA and protein levels [20]. Thus, modification of these pathways could be used as means of intervention. Although we found significant downregulation of $\mathrm{Ccl}_{2}$ in ExP, the difference between ExP and LP were not significant.

\section{Study Limitations}

There were several limitations to our study that should be explored further. We did not include an exercise only group because a large number of studies have already shown the 
A

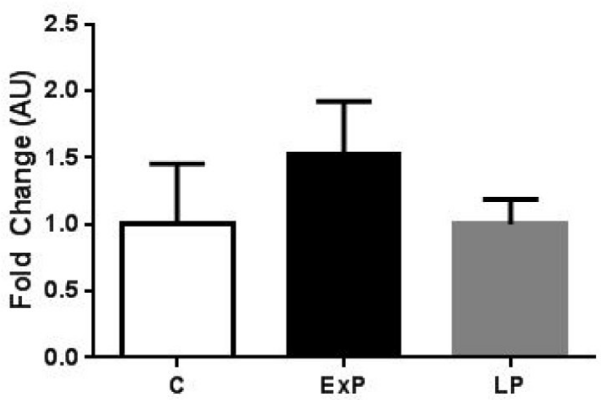

C

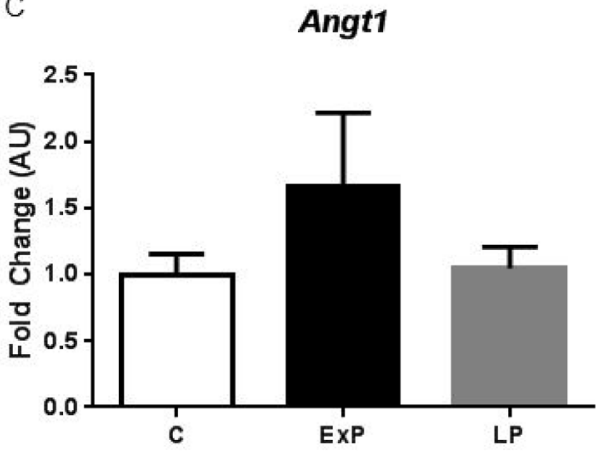

$\mathrm{E}$

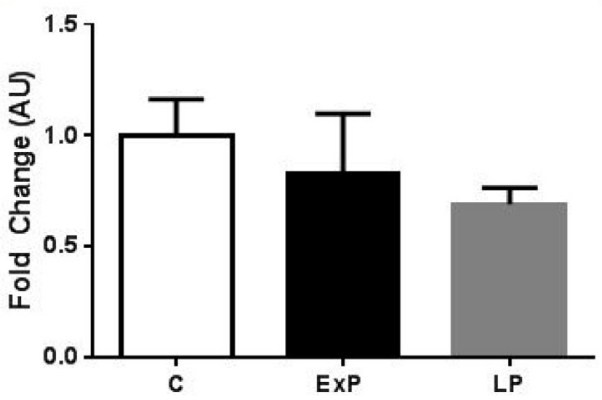

B

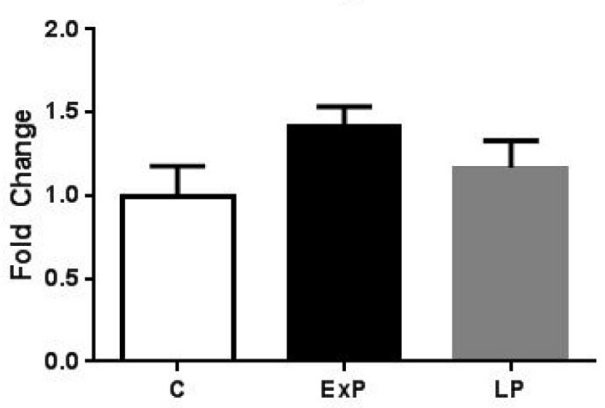

D

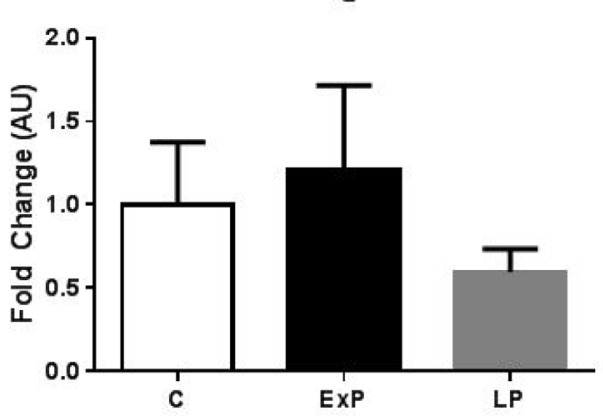

Pdgfa

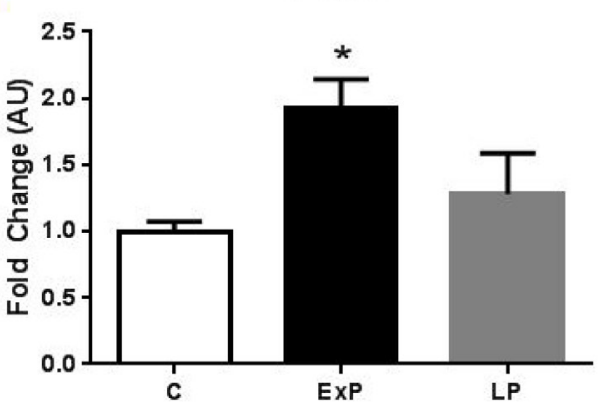

Figure 6. mRNA levels of genes regulating angiogenesis. Ppargcla: peroxisome proliferator-activated receptor gamma coactivator-1 alpha; Vegfa: vascular endothelial growth factor a; Angpt1: angiopietin-1; Angpt2: angiopietin-2; Fgf2: fibroblast growth factor 2; Pdgfa: platelet derived growth factor alpha. C: non-pregnant sedentary control; ExP: exercise during pregnancy; LP: sedentary pregnant group. Values are mean \pm SEM expressed as fold change relative to $C .{ }^{*} p<0.05$, significantly different from $C$.

molecular mechanism of exercise-induced cardiac hypertrophy. Therefore, it seemed wasteful to use mice for the information that is already known. Moreover, a previous study [22] demonstrated different gene expression profiles of cardiac hypertrophy in response to exercise-only and pregnancy-only stimulus [1]. Nonetheless, having an exercise control group may aid in making conclusions about exercise, pregnancy, and interaction effects. Next, most of the upregulated cardioprotective genes increased in the ExP as compared to the $\mathrm{C}$, but not the LP. The volume of exercise dropped off significantly at mid-pregnancy, suggesting that adding a different gestational stage, such as mid-pregnancy, should be considered in the future study to provide invaluable insight into the concurrent effects of exercise training. Alternatively, as suggested by Platt et al. [31], exercise mode that can maintain the early gestational exercise volume in late pregnancy may help to activate cardio-protective pathways rather than relying on voluntary exercise since most pregnant mice and humans do not continue to exercise voluntarily during the late stage of pregnancy [32-34]. We did not fully investigate the role of Akt and its downstream targets on cardiac hypertrophy induced by exercise during pregnancy. Using a gain of function or loss of function approach, the signaling molecules should be tested in the future study.

In summary, we demonstrate that exercise during pregnancy initiated at day one of gestation is not detrimental for the heart, but rather beneficial by activating several crucial positive genes (antioxidant- and angiogenesis- related genes), as well as downregulating a pro-inflammatory gene $\left(\mathrm{CCl}_{2}\right)$. The American College of Obstetricians and Gynecologists (ACOG) recommends that pregnant women exercise regularly, even those who were previously sedentary [35]; however, some people still perceive exercise as risky. Our results suggest that voluntary exercise can be a beneficial behavior modification during pregnancy without causing negative effects on the pregnant mother or their fetuses [21]. In addition, our study can be used by practitioners to encourage their healthy pregnant 
Citation: Chung E, Looten KD, Lunsford T, et al. Exercise during pregnancy activates cardio-protective genes without a further increase in pregnancy-induced cardiac hypertrophy. Gynecol Reproduct Endocrinol -UK. 2017;1(1):7-15

patients to participate in exercise for their own health, as well as their developing offspring. Consequently, this study has clear implications for human cardiovascular health. In a future study, it would be interesting to investigate the effects of exercise on peripartum cardiomyopathy models described previously [20], and to measure how exercise modifies the genes (i.e., Ccl2, Stat3, Sod2, and $\mathrm{Pgcla}$ ) responsible for peripartum cardiomyopathy.

\section{Acknowledgements}

This study was supported by Texas Tech University Start-Up funds to Dr. Eunhee Chung, Undergraduate Project Funding from Texas Tech University Center for Active Learning and Undergraduate Engagement (CALUE) to Kalli Looten, and the Undergraduate Research Scholar program from Texas Tech University Honors College to Taylor Lunsford.

\section{References}

1. Chung E, Leinwand LA. Pregnancy as a cardiac stress model. Cardiovasc Res. 2014;101:561-70.

2. Longo LD. Maternal blood volume and cardiac output during pregnancy: a hypothesis of endocrinologic control. Am J Physiol. 1983;245:R720-29.

3. Eghbali M, Deva R, Alioua A, et al. Molecular and functional signature of heart hypertrophy during pregnancy. Circ Res. 2005;96:1208-16.

4. Chung E, Yeung F, Leinwand LA. Akt and MAPK signaling mediate pregnancy- induced cardiac adaptation. J Appl Physiol. 2012;112:1564-75.

5. Clapp JF, Capeless E. Cardiovascular function before, during, and after the first and subsequent pregnancies. Am J Cardiol. 1997;80:1469-73.

6. Goldstein J, Sites CK, Toth MJ. Progesterone stimulates cardiac muscle protein synthesis via receptor-dependent pathway. Fertil Steril. 2004;82:430-36.

7. Weeks KL, McMullen JR. The athlete's heart vs. the failing heart: can signaling explain the two distinct outcomes? Physiology (Bethesda). 2011;26:97-105.

8. DeBosch B, Treskov I, Lupu TS, et al. Akt1 is required for physiological cardiac growth. Circulation. 2006;113:2097104.

9. McMullen JR, Shioi T, Zhang L, et al. Phosphoinositide 3-kinase(p110 \{alpha\}) plays a critical role for the induction of physiological, but not pathological, cardiac hypertrophy. Proc Natl Acad Sci. 2003;100:12355-60.

10. Hilfiker-Kleiner D, Kaminski K, Podewski E, et al. A cathepsin D-cleaved $16 \mathrm{kDa}$ form of prolactin mediates postpartum cardiomyopathy. Cell. 2007;128:589-600.

11. Konhilas JP, Maass AH, Luckey SW, et al. Sex modifies exercise and cardiac adaptation in mice. Am J Physiol Heart Circ Physiol. 2004;287:H2768-76.

12. Hilfiker-Kleiner D, Sliwa K. Pathophysiology and epidemiology of peripartum cardiomyopathy. Nat Rev Cardiol. 2014;11:364-70.
13. Roos-Hesselink JW, Ruys TPE, Stein JI, et al. Outcome of pregnancy in patients with structural or ischaemic heart disease: results of a registry of the European Society of Cardiology. Eur Heart J. 2013;34:657-65.

14. Schannwell CM, Zimmermann T, Schneppenheim M, et al. Left ventricular hypertophy and diastolic dysfunction in healthy pregnant women. Cardiology. 2002;97:73-78.

15. Li J, Umar S, Iorga A, et al. Cardiac vulnerability to ischemia/reperfusion injury drastically increases in late pregnancy. Basic Res Cardiol. 2012;107:271.

16. Patten IS, Rana S, Shahul S, et al. Cardiac angiogenic imbalance leads to peripartum cardiomyopathy. Nature. 2012;485:333-38.

17. Bellafiore M, Battaglia G, Bianco A, et al. The involvement of MMP-2 and MMP-9 in heart exercise-related angiogenesis. J Transl Med. 2013;11:283.

18. de Meirelles LR, Matsuura C, Resende Ade C, et al. Chronic exercise leads to antiaggregant, antioxidant and anti- inflammatory effects in heart failure patients. Eur J Prev Cardiol. 2014;21:1225-32.

19. Quindry J, French J, Hamilton K, et al. Exercise training provides cardioprotection against ischemia-reperfusion induced apoptosis in young and old animals. Exp Gerontol. 2005;40:416-25.

20. Ricke-Hoch M, Bultmann I, Stapel B, et al. Opposing roles of Akt and STAT3 in protection of the maternal heart from peripartum stress. Cardiovasc Res. 2014;101:587-96.

21. Chung E, Joiner HE, Skelton T, et al. Maternal exercise upregulates mitochondrial gene expression and increases enzyme activity of fetal mouse hearts. Physiological Reports. 2017;5:e13184.

22. Chung E, Heimiller J, Leinwand LA. Distinct cardiac transcriptional profiles defining pregnancy and exercise. PLoS ONE. 2012;7:e42297.

23. Chung E, Yeung F, Leinwand LA. Calcineurin activity is required for cardiac remodeling in pregnancy. Cardiovasc Res. 2013;100:402-10.

24. Eghbali M, Wang Y, Toro L, et al. Heart hypertrophy during pregnancy: a better functioning heart? Trends Cardiovasc Med. 2006;16:285-91.

25. Barger PM, Kelly DP. Fatty Acid Utilization in the Hypertrophied and Failing Heart: Molecular Regulatory Mechanisms. Am J Med Sci. 1999;318:36-42.

26. Jin H, Yang R, Li W, et al. Effects of exercise training on cardiac function, gene expression, and apoptosis in rats. Am J Physiol Heart Circ Physiol. 2000;279:H2994-3002.

27. Medeiros C, Frederico MJ, da Luz G, et al. Exercise training reduces insulin resistance and upregulates the mTOR/ p70S6k pathway in cardiac muscle of diet-induced obesity rats. J Cell Phsyiol. 2011;226:666-74.

28. Altamirano F, Oyarce C, Silva P, et al. Testosterone 
induces cardiomyocyte hypertrophy through mammalian target of rapamycin complex 1 pathway. J Endocrinol. 2009;202:299-307.

29. Iemitsu M, Miyauchi T, Maeda S, et al. Physiological and pathological cardiac hypertrophy induce different molecular phenotypes in the rat. Am J Physiol Regul Integr Comp Physiol. 2001;281:R2029-36.

30. Rimbaud S, Sanchez H, Garnier A, et al. Stimulus specific changes of energy metabolism in hypertrophied heart. J Mol Cell Cardiol. 2009;46:952-59.

31. Platt KM, Charnigo RJ, Kincer JF, et al. Controlled exercise is a safe pregnancy intervention in mice. J Am Assoc Lab Anim Sci. 2013;52:524-30.

32. Gilbert JS, Banek CT, Bauer AJ, et al. Placental and vascular adaptations to exercise training before and during pregnancy in the rat. Am J Physiol Regul Integr Comp Physiol. 2012;303:R520-R6.

33. Carter LG, Qi NR, Cabo RD, et al. Maternal exercise improves insulin sensitivity in mature rat offspring. Med Sci Sports Exerc. 2013;45:832-40.

34. Kim Y, Chung E. Descriptive Epidemiology of Objectively Measured Walking Among US Pregnant Women: National Health and Nutrition Examination Survey, 2005- 2006. Preventing Chronic Disease. 2015;12:E217.

35. Artal R, O'Toole M. Guidelines of the American College of Obstetricians and Gynecologists for exercise during pregnancy and the postpartum period. Br J Sports Med. 2003;37:6-12.

\section{*Correspondence to:}

Eunhee Chung

Department of Kinesiology

Health and Nutrition

University of Texas

San Antonio

TX, USA

Tel.: + 1 210-458-6723

Fax: + 1 210-458-5873

E-mail: eunhee.chung@utsa.edu 